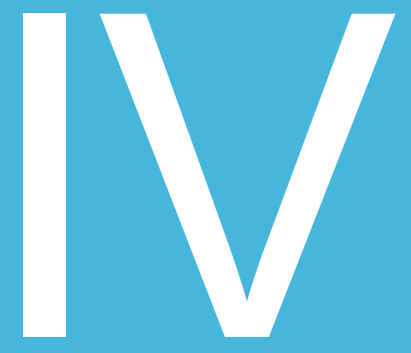

\title{
Razones que inciden en la elección de la universidad en estudiantes del nivel secundario de la Región San Martín
}

Reasons that influence the choice of an University in secondary students of the Region San Martin

Universidad Peruana Unión, Perú

\section{José Tarrillo}

Ingeniero comercial por la Universidad Adventista de Chile. Líder del grupo de investigación Target. Docente asociado de la Universidad Peruana Unión. 


\section{Resumen}

El objetivo del presente estudio fue identificar las razones que inciden en la elección de la universidad en estudiantes del nivel secundario de la Región San Martín. La muestra de estudio estuvo compuesta por 1528 escolares del nivel secundario, ambos sexos entre edades de 14 y 19 años. Se construyó un cuestionario que mide las razones para elegir una universidad. Se tomaron en cuenta las siguientes razones: Nivel académico, Precios de las Carreras, Ubicación física del Campus, Ambiente Estudiantil y Convenios Laborales. Las propiedades psicométricas evidencian que es válido y confiable. Entre los principales hallazgos se encontró que el $72.2 \%$ de escolares de secundaria, elegiría una universidad por el nivel académico, el $11.2 \%$ por los convenios laborales y un $7.7 \%$ por los precios de las carreras.

Palabras clave: Estudiante universitario, perfil universitario, carreras universitarias, educación superior.

\section{Abstract}

The aim of this study was to identify the reasons that influence the choice of an University in secondary students of the Region San Martin. The study sample consisted of 1528 secondary students, both sexes between 14 and 19 years. A questionnaire that measures the reasons for choosing a university was built. Academic level, prices of Carreras, physical location Campus, Student Environment and Labor Conventions: The following reasons were taken into account. The psychometric properties show that it is valid and reliable. Among the main findings it was found that $72.2 \%$ of secondary school, choose a university for the academic level, $11.2 \%$ for labor agreements and $7.7 \%$ for the prices of degrees.

Keywords: College student, university profile, university degrees, higher education. 


\section{Introducción}

La educación superior es una oportunidad de contribución al país y formación personal por ello es importante tener la información necesaria para tomar una decisión de la Universidad donde estudiar. Esta decisión se ha convertido en factor clave a la hora de elegir las distintas casas de estudio superior en la región San Martin. Se considera que el ser humano toma tres decisiones de vital importancia en su vida: ¿a qué se va dedicar profesionalmente? ¿Con quién contraer matrimonio? Y ¿a dónde se va ir cuando deje esta vida? Luna (2008). Sin duda que la primera pregunta se relaciona con la definición del oficio que desempeñará esa persona y esto definitivamente puede involucrar la decisión de elegir una universidad para realizar algún estudio de nivel superior, escoger una universidad es una de las decisiones más importantes que debe tomar quien desee estudiar una carrera, el tema ha llamado la atención de académicos, que han analizado el problema desde diferentes enfoques, y han discutido las ventajas y desventajas de considerar ciertas razones en el análisis, los que son muchos y muy variados.

Muchos alumnos declaran que en el proceso de selección de una universidad integran solamente razones de tipo económico y que ello les evita frustraciones al tener que desertar de la Universidad por falta de recursos Beswick (1989). Por su parte, Veloutsou, Patón y Lewis (2005) señalan que muchos estudiantes incluyen en la evaluación de las diferentes razones solamente los atributos o factores que les proporcionan las universidades y que generalmente no se apoyan en las consejerías de orientación vocacional; y si bien los alumnos reportaron satisfacción con la información proporcionada por dichas instituciones, Ballinger (2005) señala que esta elección debe ser una experiencia académica relevante y divertida, por lo que no existe razón para temer investigar información adicional relacionada con las universidades que les interesen, lo cual se realiza fácilmente en la actualidad a través de las páginas web de las Universidades. Dado que la decisión de elegir una universidad no es una responsabilidad solamente del futuro alumno, Warwick y Mansfield (2003) recomiendan que dicha decisión incluya a los padres, quienes tienen más experiencia y capacidad de análisis. Afortunadamente, como menciona Hartman (1997) y Jones (2011), en la actualidad existen muchos recursos en Internet que pueden ser consultados desde cualquier parte del mundo, evitando así la omisión de razones importantes.

Asimismo, las razones presentadas en este estudio se determinaron de la entrevista que se hizo a un grupo de alumnos de nivel secundario quienes mencionaron sus razones para elegir una Universidad de los cuales 
se consideraron la siguientes razones para elegir una Universidad: el aspecto económico es uno de los principales factores a considerar, ya que es citado por nueve de las once fuentes consultadas; el segundo lugar lo ocupa la ubicación física del campus en la que se encuentra establecida la universidad, con ocho recomendaciones de las once fuentes. Otro factor que es citado ampliamente es los precios de las carreras que cuenta la universidad, seguido del Ambiente estudiantil de institución (pública o privada), así como los convenios laborales que tenga la Universidad. (Entrevista realizada 1 al 15 abril 2016).

De esta información se escogieron las cinco razones presentadas a los estudiantes secundarios como son: nivel académico ,precios de las carreras, ubicación física del campus, ambiente estudiantil, convenios laborales; permitiendo a los estudiantes contar con mejor información para elegir una Universidad para realizar sus estudios universitarios, es una labor difícil en la cual el adolescente se encuentra en una encrucijada y en la literatura sólo existen reportes en los que se indica lo que se debe considerar al momento de tomar una decisión de ese tipo, por lo que no pasan de ser solamente recomendaciones; hay una carencia de estudios de tipo cuantitativo que surjan de bases empíricas e indiquen cuáles son las verdaderas razones que los secundarios toman en cuenta al realizar la selección de una Universidad para estudiar.

Así pues, la pregunta que cabe hacer es: ¿cuáles son las razones que los alumnos secundarios de la región san Martin consideran para elegir la universidad donde estudiar? y ¿̇uál es el nivel de importancia que se le proporciona a estas razones? Para responder a las preguntas anteriores, el objetivo de este artículo es presentar los resultados de un estudio realizado en once instituciones de educación secundaria de la región san Martin, el cual estaba encaminado a determinar el nivel de importancia que los alumnos daban a las cinco razones planteadas que los alumnos consideren al tomar la decisión de formar parte de una universidad.

\section{Materiales y Métodos}

La investigación corresponde a un diseño no-experimental, de tipo descriptivo porque se buscó determinar las razones que inciden en la elección de la universidad, en los alumnos del nivel secundario de la Región San Martin. (Hernández, Fernández y Baptista, 2010).

\section{Participantes}

La muestra del presente estudio estuvo compuesta por 1528 estudiantes (799 varones y 781 mujeres) con edades comprendidas entre los 14 y 19 años. 
Estos estudiantes se encontraban matriculados en Instituciones Educativas Privadas y Estatales de la Región San Martín, Perú (Tarapoto, Lamas, Tocache, Juan Guerra, Cacatachi)

Se aprecia en la tabla 1 que el $81.9 \%$ de escolares oscila entre $14-15$ años de edad. Por otro lado, el $50.6 \%$ de participantes fueron mujeres y el $49.4 \%$ hombres. En cuanto al grado, se aprecia que el $73.9 \%$ se encuentra en $4^{\circ}$ y $5^{\circ}$ grado de secundaria. $Y$ el mayor porcentaje de estudiantes participantes son de la Institución Educativa Ofelia Velásquez (37.5\%).

Tabla 1

Características sociodemográficas de los escolares

\begin{tabular}{|c|c|c|c|}
\hline \multicolumn{2}{|r|}{ Variables } & \multirow{2}{*}{$\begin{array}{c}\mathbf{n} \\
537\end{array}$} & \multirow{2}{*}{$\begin{array}{c}\% \\
40.2\end{array}$} \\
\hline Edad & 14 años & & \\
\hline & 15 años & 545 & 41.7 \\
\hline & $\geq 16$ años & 528 & 18.1 \\
\hline Sexo & Femenino & 781 & 49.4 \\
\hline \multirow{4}{*}{ Grado } & Masculino & 799 & 50.6 \\
\hline & $3^{\circ}$ secundaria & 412 & 26.1 \\
\hline & $4^{\circ}$ secundaria & 542 & 34.3 \\
\hline & $5^{\circ}$ secundaria & 626 & 39.6 \\
\hline \multirow{11}{*}{$\begin{array}{l}\text { Institución } \\
\text { Educativa }\end{array}$} & I.E Aplicación & 190 & 12.0 \\
\hline & I.E José María Arguedas-Cacatachi & 95 & 6.0 \\
\hline & I.E María Ulises Reátegui Pinedo & 97 & 6.1 \\
\hline & I.E Ofelia Velásquez & 592 & 37.5 \\
\hline & I.E Tarapoto & 130 & 8.2 \\
\hline & I.E. Juan Guerra & 116 & 7.3 \\
\hline & I.E. San Lucas & 18 & 1.1 \\
\hline & I.E.P Ciencias & 128 & 8.1 \\
\hline & I.E.P Virgen de las mercedes & 13 & .8 \\
\hline & I.E.P. José de San Martín & 79 & 5.0 \\
\hline & Lamas 0255 & 122 & 7.7 \\
\hline
\end{tabular}

Total $=1528$ escolares

\section{Instrumentos}

Para la evaluación de las razones que indicen en la elección de la universidad, se aplicó un cuestionario en donde se plantearon las preguntas que sirvieron como guía para indagar sobre determinadas razones de elección. 
Para validar el instrumento se solicitó el juicio de expertos, quienes evaluaron la coherencia, claridad, suficiencia, cumplimiento de objetivos, replicabilidad y relevancia de los ítems presentado en el cuestionario.

\section{Análisis de datos}

Para el procesamiento y análisis de datos, se utilizó el paquete estadístico SPSS versión 20 para Windows, aplicado a las ciencias sociales; también, se utilizó técnicas estadísticas descriptivas a un nivel de significancia $p<0.05$.

\section{Resultados}

Resultados del análisis descriptivo de las razones que inciden en la elección de la universidad

La Tabla 2 muestra que el $72.2 \%$ de escolares, elegiría una universidad por el nivel académico, el $11.2 \%$ por los convenios laborales y un $7.7 \%$ por los precios de las carreras, siendo estos datos importantes en la presente investigación.

Tabla 2

Razones que inciden en la elección de universidad en estudiantes del nivel secundario de la Región San Martín

\begin{tabular}{lcc}
\hline & Frecuencia & Porcentaje \\
\hline Nivel Académico & 1141 & $72.2 \%$ \\
Precios de las carreras & 122 & $7.7 \%$ \\
Ubicación Física del & 52 & $3.3 \%$ \\
Campus & & \\
Ambiente Estudiantil & 88 & $5.6 \%$ \\
Convenios Laborales & 177 & $11.2 \%$ \\
Total & 1580 & $100.0 \%$ \\
\hline
\end{tabular}

La Tabla 3 muestra que los hombres y las mujeres elegirían una universidad por el nivel académico ( $34.6 \%$ y $37.7 \%$ respectivamente). Asimismo se aprecia que el $6.7 \%$ de hombres y el $4.5 \%$ de mujeres elegiría estudiar en una universidad por los convenios laborales, de manera similar ocurre con los precios de las carreras ( $4.1 \%$ y $3.6 \%$ respectivamente). 
Razones que inciden en la elección de la universidad en estudiantes

Tabla 3

Razones que inciden en la elección de universidad en estudiantes del nivel secundario de la Región San Martín, según genero

\begin{tabular}{llcccc}
\hline & & \multicolumn{3}{c}{ Genero } \\
\cline { 3 - 6 } & & \multicolumn{2}{c}{ Femenino } & \multicolumn{2}{c}{ Masculino } \\
\hline Razones & Nivel Académico & 595 & $37.7 \%$ & 546 & $34.6 \%$ \\
para elegir & Precios de las carreras & 57 & $3.6 \%$ & 65 & $4.1 \%$ \\
una Univer- & Ubicación Física del & 19 & $1.2 \%$ & 33 & $2.1 \%$ \\
sidad & Campus & & & & \\
& Ambiente Estudiantil & 39 & $2.5 \%$ & 49 & $3.1 \%$ \\
& Convenios Laborales & 71 & $4.5 \%$ & 106 & $6.7 \%$ \\
\cline { 2 - 6 } & Total & 781 & $49.4 \%$ & 799 & $50.6 \%$ \\
\hline
\end{tabular}

En la tabla 4 se analiza que los escolares del nivel secundario de la Región San Martín, respecto a las edades consideradas en el estudio; elegirían una universidad por el nivel académico (25.7\%, 25.9\%, $18.1 \%, 2 \%, .4 \%$ y .1\% respectivamente).

\section{Tabla 4}

Razones que inciden en la elección de universidad en estudiantes del nivel secundario de la Región San Martin, según edad

\begin{tabular}{|c|c|c|c|c|c|c|c|c|c|c|c|c|c|}
\hline \multirow{4}{*}{$\begin{array}{l}\text { Razo- } \\
\text { nes } \\
\text { para } \\
\text { elegir } \\
\text { una } \\
\text { Univer- } \\
\text { sidad }\end{array}$} & \multirow[b]{3}{*}{$\begin{array}{l}\text { Nivel Aca- } \\
\text { démico }\end{array}$} & \multicolumn{12}{|c|}{ Edad } \\
\hline & & \multicolumn{2}{|c|}{14} & \multicolumn{2}{|r|}{15} & \multicolumn{2}{|c|}{16} & \multicolumn{2}{|c|}{17} & \multicolumn{2}{|c|}{18} & \multicolumn{2}{|r|}{19} \\
\hline & & 406 & $25.7 \%$ & 409 & $25.9 \%$ & 286 & $18.1 \%$ & 31 & $2.0 \%$ & 7 & $.4 \%$ & 2 & $.1 \%$ \\
\hline & $\begin{array}{l}\text { Precios de } \\
\text { las carre- } \\
\text { ras }\end{array}$ & 39 & $2.5 \%$ & 40 & $2.5 \%$ & 27 & $1.7 \%$ & 11 & $.7 \%$ & 5 & $.3 \%$ & 0 & $0.0 \%$ \\
\hline sidad & $\begin{array}{l}\text { Ubicación } \\
\text { Física del } \\
\text { Campus }\end{array}$ & 13 & $.8 \%$ & 20 & $1.3 \%$ & 18 & $1.1 \%$ & 0 & $0.0 \%$ & 1 & $.1 \%$ & 0 & $0.0 \%$ \\
\hline & $\begin{array}{l}\text { Ambiente } \\
\text { Estudiantil }\end{array}$ & 31 & $2.0 \%$ & 31 & $2.0 \%$ & 19 & $1.2 \%$ & 5 & $.3 \%$ & 1 & $.1 \%$ & 1 & $.1 \%$ \\
\hline & $\begin{array}{l}\text { Convenios } \\
\text { Laborales }\end{array}$ & 48 & $3.0 \%$ & 45 & $2.8 \%$ & 59 & $3.7 \%$ & 21 & $1.3 \%$ & 3 & $.2 \%$ & 1 & $.1 \%$ \\
\hline & Total & 537 & $34.0 \%$ & 545 & $34.5 \%$ & 409 & $25.9 \%$ & 68 & $4.3 \%$ & 17 & $1.1 \%$ & 4 & $.3 \%$ \\
\hline
\end{tabular}

Se aprecia en la Tabla 5, que el 33.9\% de los escolares encuestados, le gustaría estudiar en la Universidad Nacional de San Martín, un 30.3\% en la Universidad Cesar Vallejo y un $16.4 \%$ en la Universidad Peruana Unión.

Revista Apunt. Univ.

VOLUMEN VI • NÚMERO 2 p. 69 - 79 
José Tarrillo

Tabla 5

Universidades donde le gustaría estudiar a los escolares de la Región San Martín

\begin{tabular}{|c|c|c|c|}
\hline \multirow{20}{*}{$\begin{array}{l}\text { Universidades } \\
\text { donde le gusta- } \\
\text { ría estudiar }\end{array}$} & & Frecuencia & Porcentaje \\
\hline & CATÓLICA & 29 & 1.8 \\
\hline & SAN MARCOS & 47 & 3.0 \\
\hline & UAP & 132 & 8.4 \\
\hline & UCP & 49 & 3.1 \\
\hline & UCV & 479 & 30.3 \\
\hline & UIGV & 1 & .1 \\
\hline & UNAS & 1 & .1 \\
\hline & UNI & 22 & 1.4 \\
\hline & UNSM & 535 & 33.9 \\
\hline & UNSMP & 1 & .1 \\
\hline & UNT & 3 & .2 \\
\hline & UPAO & 5 & .3 \\
\hline & UPC & 6 & .4 \\
\hline & UPEU & 259 & 16.4 \\
\hline & UPN & 1 & .1 \\
\hline & USIL & 7 & .4 \\
\hline & USMP & 1 & .1 \\
\hline & UTP & 2 & .1 \\
\hline & Total & 1580 & 100.0 \\
\hline
\end{tabular}

La Tabla 6 muestra que tanto a hombres y mujeres les gustaría estudiar en la Universidad Nacional San Martín (15.5\% y $18.4 \%$ respectivamente). Asimismo se aprecia que el $16.4 \%$ de hombres y el $13.9 \%$ de mujeres le gustaría estudiar en el Universidad Cesar Vallejo y al $8.6 \%$ de mujeres y $7.8 \%$ de varones les gustaría estudiar en la Universidad Peruana Unión. 
Razones que inciden en la elección de la universidad en estudiantes

Tabla 6

Universidades donde le gustaría estudiar a los escolares de la Región San Martín, según genero

\begin{tabular}{llcccc}
\hline & & \multicolumn{4}{c}{ Genero } \\
\cline { 3 - 6 } & CATÓLICA & 17 & $1.1 \%$ & 12 & $.8 \%$ \\
& SAN MARCOS & 21 & $1.3 \%$ & 26 & $1.6 \%$ \\
& UAP & 61 & $3.9 \%$ & 71 & $4.5 \%$ \\
& UCP & 29 & $1.8 \%$ & 20 & $1.3 \%$ \\
& UCV & 220 & $13.9 \%$ & 259 & $16.4 \%$ \\
Priorice las & UIGV & 1 & $.1 \%$ & 0 & $0.0 \%$ \\
Universidades & UNSM & 0 & $0.0 \%$ & 1 & $.1 \%$ \\
donde le gus- & UNSMP & 7 & $.4 \%$ & 15 & $.9 \%$ \\
taría estudiar & UNT & 290 & $18.4 \%$ & 245 & $15.5 \%$ \\
& UNI & 0 & $0.0 \%$ & 1 & $.1 \%$ \\
& UPAO & 2 & $.1 \%$ & 1 & $.1 \%$ \\
& UPC & 3 & $.2 \%$ & 2 & $.1 \%$ \\
& UPEU & 3 & $.2 \%$ & 3 & $.2 \%$ \\
& UPN & 123 & $7.8 \%$ & 136 & $8.6 \%$ \\
& USIL & 0 & $0.0 \%$ & 1 & $.1 \%$ \\
& USMP & 2 & $.1 \%$ & 5 & $.3 \%$ \\
& UTP & 0 & $0.0 \%$ & 1 & $.1 \%$ \\
& Total & 2 & $.1 \%$ & 0 & $0.0 \%$ \\
& & 781 & $49.4 \%$ & 799 & $50.6 \%$ \\
\hline
\end{tabular}

\section{Discusión}

El objetivo del estudio fue determinar las razones que inciden al momento de escoger una universidad y cuál de ellas tienen mayor injerencia en los jóvenes secundarios. Se encontró que los escolares eligen una universidad por el nivel académico con un índice de $72.2 \%$. Datos similares se aprecian en el estudio realizado por Alcaraz et al (2006) donde el nivel académico es el segundo factor de mayor injerencia al momento de escoger una universidad. El $50.6 \%$ de participantes fueron mujeres y el $49.4 \%$ hombres. En cuanto al grado, se aprecia que el $73.9 \%$ se encuentra en $4^{\circ}$ y $5^{\circ}$ grado de secundaria.

Respecto a las diferencias por sexo, se encontró que las participantes mujeres obtuvieron puntajes más altos en la razón de nivel académico con $37.7 \%$, es decir eligen en base al nivel académico. Respecto a las edades consideradas en el estudio los alumnos de 14 a 15 años; eligen una universidad por el nivel académico lo que representa un $51.6 \%$. Asimismo, se encontró que 
tanto a hombres como mujeres les gustaría estudiar en la universidad nacional ( $15.5 \%$ y $18.4 \%$ respectivamente). Asimismo se aprecia que el $16.4 \%$ de hombres y el $13.9 \%$ de mujeres le gustaría estudiar en instituciones privadas y el $8.6 \%$ de mujeres y $7.8 \%$ de varones les gustaría estudiar en la Universidad Peruana Unión, esto está en consonancia con la percepción del alto nivel académico de Is instituciones superiores nacionales.

La muestra estuvo compuesta por 1528 estudiantes (799 varones y 781 mujeres) con edades comprendidas entre los 14 y 19 años. Estos estudiantes se encontraban matriculados en instituciones educativas privadas y estatales de la Región San Martín, Perú (Tarapoto, Lamas, Tocache, Juan Guerra, Cacatachi). Respecto a las limitaciones, la investigación destaca que sólo se consideraron estudiantes de $3^{\circ}, 4^{\circ}$ y $5^{\circ}$ grado de secundaria y de algunas ciudades de la Región San Martín, por lo que es importante ampliar el estudio a otras ciudades de la región.

Asimismo el presente estudio sugiere investigaciones longitudinales que permitan examinar las razones que llevaron a escoger una universidad y una carrera profesional. Asimismo, otra razón significativa identificada en este estudio es el de los convenios laborales de la universidad, es importante que la universidades cuenten con alianzas o convenios laborales con las principales empresas de la región que permitan al estudiante secundario visualizar un trabajo al concluir sus estudios en la Universidad, esto concuerda con el estudio de Schweitzer (2009), donde señala que los estudiantes secundarios eligen las universidades americanas que cuentan con convenios laborales para todas las carreras.

Otra de las razones que resultó importante en el análisis es las mensualidades de las carreras que tiene la universidad; la mayoría de los estudiantes se desempeñan simultáneamente en trabajos de agricultura y comercio. Este fenómeno ha sido identificado por Vrontis et al. (2007), quien señala que los estudiantes en la actualidad buscan independencia económica y comparten su tiempo entre el trabajo y la Universidad, por lo que una razón importante al momento de escoger una universidad son los precios de sus carreras.

Se concluye que los jóvenes consideran que las universidades existentes en la región deben invertir más en cuidar y mejorar su nivel académico esto significa incentivar la investigación, publicación y visibilizarlo a la comunidad, los convenios laborales deben ser una exigencia de toda universidad, deben trabajar por ampliar sus convenios laborales no solo a nivel local sino nacional y extranjero, de esa manera la universidad será un atractivo para los alumnos 
secundarios, también es importante que las universidades puedan asimilar la importancia que tienen los precios de las carreras considerando que un buen porcentaje de alumnos que termina la secundaria no cuenta con apoyo de sus padres, deben estudiar y buscar ese equilibrio en los precios de las carreras que permitan la inclusión de más jóvenes a la educación superior.

La mejora de la calidad académica, las oportunidades a través de los convenios laborales y el nivel de los precios de sus carreras constituyen razones de exigencia por parte de los estudiantes encuestados. El éxito del crecimiento en toda empresa es saber escuchar a nuestros clientes, y mejorar lo que ellos nos piden mejorar.

José Tarrillo Paredes Universidad Peruana Unión, Perú email: jose.tarrillo@upeu.edu.pe

Recibido: 17 de febrero de 2016 Aceptado: 14 de marzo de 2016

\section{Referencias}

Caccuri, V. (2008). "Elegir la universidad por Internet", estudiantes.org Disponible en: http://www. estudiante.org/elegir-la-universidad-por-internet/ (consultado 8 de mayo de 2016).

Carrasco, J. A. (2007). “La educación quirúrgica: nuevos paradigmas”, Cirugía y Cirujanos, vol. 75, núm. I, pp. 1-2.

Covey, S. (2010). Las 6 decisiones más importantes de tu vida, Ciudad de México: Grijalvo.

Espinoza, O. y González, L. E. (2008). "Perfil socioeconómico del estudiantado que accede a la educación superior en Chile (1990-2003)”, Estudios Pedagógicos, vol. 34, núm. I, pp. 27-39.

García, A; Gutiérrez, M. T; Gómez, J; Pérez, V. y Baque, E. F. (2006). “¿Qué es la Psicología para los estudiantes españoles de educación a distancia?" Revista Latinoamericana de Psicología, vol. 38, núm. 2, pp. 383-396.

Luna, J. D. (2008). “Las 3 decisiones más importantes de la vida”, en Jd Luna's Disponible en: http:// juandiegoluna.com/2008/05/07/decisionesdelavida/ (consultado 6 de mayo de 2016).

Monjo-Sacristán, R. (2009). “¿Qué criterios debes tener en cuenta a la hora de elegir universidad?”, en educaweb.com. Disponible en: http://www.educaweb.com/noticia/2009/05/18/ criterios-debes-tener-cuenta-hora-elegir-universidad-136 58.html (consultado el 1 de junio de 2016).

Pascual, I. R. (1999). Consejos para elegir su universidad privada, Madrid: El Mundo.

Schuldt, M. (2011). Diez factores a considerar al elegir una universidad, Chicago: College Admissions Services, Inc.

Yaq, P. (2010). “Cómo elegir una universidad a tu medida, sin estresarte (icasi!)", en Yaq.es. Decidiendo qué y cómo estudiar, Madrid. Disponible en: http://www.yaq.es/reportajes/comoelegir-universidad (consultado 8 de mayo de 2016). 